\title{
Chaos Communication Performance: Theory and Computation
}

\author{
G Kaddoum, A J Lawrance, P Chargé, and D Roviras
}

\begin{abstract}
In this paper new and existing approaches are developed to compute the bit error rate for chaos-based communication systems. The multi-user coherent antipodal chaos shift keying system is studied and evaluated in its coherent form, in the sense of perfect synchronization between transmitted and received chaotic sequences. Transmission is through an additive white Gaussian noise channel. Four methods are interrelated in the paper, three approximate ones and an exact one. The least accurate but most well known is based on simple Gaussian approximation; this is generalized to better reveal its structure. Two accurate and computationally efficient approximate methods are based on conditional Gaussian approximation and the statistical distribution of the typically non-constant bit energy. The most insightful but computationally expensive one is based on exact theory and rests on explicit mathematical results for particular chaotic maps used to spread binary messages. Both upper and lower bounds to the bit error rate are suggested. The relative advantages of the different approaches are illustrated with plots of bit error rate against signal to noise ratio.
\end{abstract}

\section{Key Words}

Bit energy, Bit error, Chaos shift keying communication, Exact and Gaussian approximations, Multi-user

Author's accepted manuscript of the article published in

Circuits, Systems, and Signal Processing, vol. 30, n० 1 (2011)

The final publication is available at link.springer.com

http://dx.doi.org/10.1007/s00034-010-9217-1 


\section{Introduction}

The chaotic signal is a relatively new field in communication systems. Motivation derives from the advantages offered by chaotic signals, such as spread spectrum, robustness in multipath environments and resistance to jamming. Chaotic signals are non-periodic, broadband, and difficult to predict and to reconstruct; they may be generated by mathematical map functions, electronic circuits or laser optics. Their properties coincide with requirements for signals used in communication systems, in particular for spreadspectrum communication and secure communication systems. The possibilities to generate an infinite number of sequences make for easy modelling and application of these signals in the single and multi-user cases. A wide overview of the general area can be obtained from the monographs [9], [15], [23], [14] and [27], although not yet reaching work in laser optics [28], [2]. The paper focuses on the multi-user chaos shift-keying system of communication with chaotic map spreading, emerging from [6], [11], [12] and [24]. The main concern here is the theory and computation of bit error rate (BER), the primary measure of performance for many types of system. The three approximate methods for its calculation are considered, simple Gaussian approximation (SGA), which is inaccurate but indicative of the main influences on bit error and two forms of conditional Gaussian approximation, CGA-Simulation (CGA-S) and CGA-Analytical (CGA-A), which can be very accurate. These are followed by emphasis on an exact Gaussian theory (EGT) method which is insightful and either perfectly exact or nearly exact, but is much more demanding in computational terms. The paper thus sets out the choices of approximation and exact results, with illustrations.

The organization of the paper is as follows. Section 2 introduces multi-user CSK systems and their correlator demodulation. Sections 3 and 4 give accounts of SGA and CGA which newly emphasize the effect of autocorrelation in chaotic spreading on BER. Section 5 mentions three differing chaotic maps and their bit energy distributions. Section 6 links bit energy and CGA to give a very effective simulation-based method of calculating BER, CGA-Simulation. This method is further developed in Section 7 to the CGA-Analytical method which avoids numerical integration in obtaining BER. In Sections 8-9 EGT methods are newly developed for multi-user systems, allowing insights computations for the effects of multi-users and autocorrelated spreading on BER computations. Finally Section 10 shows that a previously developed likelihood-based generalized correlation decoder is effective in reducing BER in multi-user systems.

\section{Multi-user Antipodal CSK Communication}

In this section, the multi-user antipodal coherent chaos-shift-keying (CSK) communication system is described. A block diagram of this system is shown in Fig. 1.

\section{CSK Transmission scheme}

The CSK system in Fig. 1 has $L$ users. A stream of binary bit data symbols from active-user $l\left(b_{l, i}= \pm 1, i=1,2, \ldots\right)$ with bit period $T_{b}$ is spread by a chaotic signal generated at the transmitter. Symbol bits of different users are independent of each another. The spreading factor $(N)$ is the number of chaotic samples in a bit duration and these constitute a chaotic segment; $T_{c}$ is the time between each chaotic sample (chip), so $T_{b}=N T_{c}$. 
The chaotic segments of all users are generated by the same chaotic map $\tau(\cdot)$, of specified form. Early communication uses of such maps include [10] and [4]. For the $l^{\text {th }}$ user, the chaotic segment $\left\{x_{l}\right\}$ is assumed to have been started with a random initial sample value $x_{l, 0}$ from the natural invariant distribution of $\tau(\cdot)$. As random variables, the chaotic samples of the $l^{\text {th }}$ spreading segment are assumed to have zero mean which has no effect on the dynamical properties, and the common variance is denoted by $\sigma_{X}^{2}$. The output of the chaotic signal generator used by the $l^{\text {th }}$ user is thus given by

$$
u_{l}(t)=\sum_{i=0}^{N-1} x_{l, i} g_{T_{c}}\left(t-i T_{c}\right)
$$

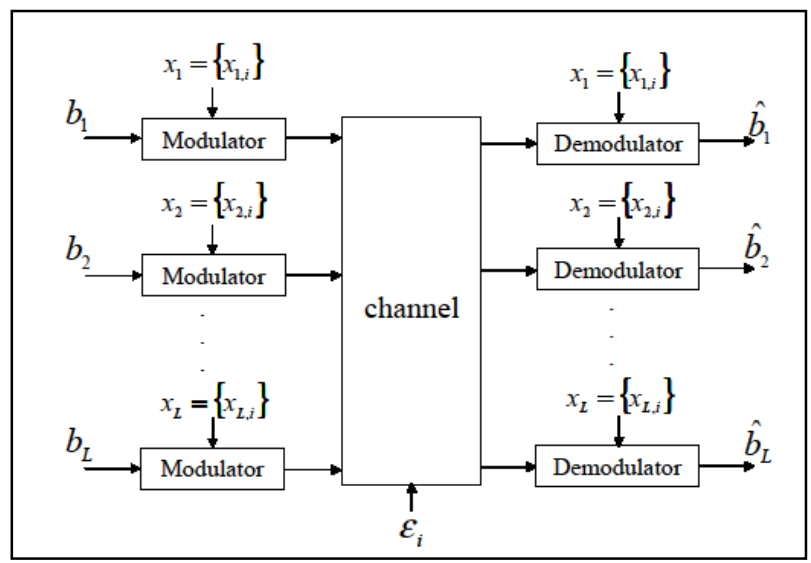

Fig. 1. Block diagram of a multi-user coherent antipodal CSK communication system.

The function $g_{T_{c}}(t)$ is a rectangular pulse of unit amplitude and width $T_{c}$ given by

$$
g_{T_{c}}(t)= \begin{cases}1 & 0 \leq t \leq T_{c} \\ 0 & \text { elsewhere. }\end{cases}
$$

In the antipodal CSK system, the modulation of a typical bit $b_{l}= \pm 1$, say the $j^{\text {th }}$ in the bit stream of the $l^{\text {th }}$ user, takes the form $b_{l} x_{l}$. The transmitted signal of the $l^{\text {th }}$ user is thus

$$
s_{l}(t)=\sum_{i=0}^{N-1}\left(b_{l} x_{l, i}\right) g_{T_{c}}\left(t-i T_{c}\right) .
$$

The energy of a typical bit of the $l^{\text {th }}$ user is given by

$$
E_{b c}^{(l)}=T_{c} \sum_{i=0}^{N-1} x_{l, i}^{2}
$$

and is thus seen not to be constant, in contrast to conventional systems, such as binary phase shift-keying (BPSK).

\section{Channel model}

An additive white Gaussian noise (AWGN) channel is assumed; $n(t)$ is the Gaussian noise affecting reception by the $l^{\text {th }}$ user and has a two-sided power spectral density given 
by

$$
S_{n}(f)=N_{0} / 2
$$

Let $n_{t}^{\prime}(t)$ be the equivalent noise source of $n_{l}(t)$ specified as

$$
n^{\prime}(t)=\sum_{i=0}^{\infty} \varepsilon_{l, i} g_{T_{c}}\left(t-i T_{c}\right)
$$

where $\left\{\varepsilon_{l, i}\right\}$ are independent Gaussian random variables with zero mean and variance

$$
\sigma_{n}^{2}=N_{0} / 2 T_{c}
$$

The channel is taken as flat and slow fading for all users; therefore all frequency components of the signal will experience the same magnitude of fading. This means that amplitude and phase changes imposed by the channel can be considered reasonably constant over a long sequence of transmitted symbols. Furthermore, all users are asynchronous and the received signal $r_{l}(t)$ of user $l$ at time $t$ includes the sum of $L-1$ interference signals from the other users and the system's AWG noise, and so is given by

$$
r_{l}(t)=s_{l}(t)+\sum_{k=1, k \neq l}^{L} s_{k}(t)+n^{\prime}(t) .
$$

By substituting (3) and (6) into (8) there is

$$
\begin{aligned}
r_{l}(t) & =\sum_{i=0}^{N-1}\left\{b_{l} x_{l, i}+\sum_{k=1, k \neq l}^{L} b_{k} x_{k, i}+\varepsilon_{i}\right\} g_{T_{c}}\left(t-i T_{c}\right) \\
& =\sum_{i=0}^{N-1} z_{l, i} g_{T_{c}}\left(t-i T_{c}\right)
\end{aligned}
$$

in terms of the received samples $z_{l, i}$ of a typical bit of the $l^{\text {th }}$ user, where

$$
z_{l, i}=b_{l} x_{l, i}+\sum_{k=1, k \neq l}^{L} b_{k} x_{k, i}+\varepsilon_{i}, i=0,1, \ldots, N-1
$$

This can be written more concisely as

$$
z_{l}=b_{l} x_{l}+\sum_{k=1, \neq l}^{L} b_{k} x_{l}+\varepsilon
$$

where $z_{l}, x_{l}$ and $\varepsilon$ are column vectors with entries $z_{l, i}, x_{l, i}$ and $\varepsilon_{i}, i=0,1, \ldots, N-1$, respectively.

\section{CSK Demodulation scheme}

In coherent CSK communication systems perfect synchronization is assumed at the receiver side. The demodulator can reproduce a reference sequence which is the exact replica of the chaotic samples used at the transmitter to spread the bit information. In order to demodulate the transmitted signal of the $l^{\text {th }}$ user, the $l^{\text {th }}$ receiver only knows the $l^{\text {th }}$ chaotic reference sequence, $u_{l}(t)$, and no information from of the other users. 
The conventional correlator, used as a demodulator, takes the form

$$
C_{r, u}=\int_{0}^{N T_{c}} r_{l}(t) u_{l}(t) d t
$$

By placing (1) and (9) in (12), the correlator becomes

$$
C\left(z_{l}, x_{l}\right)=T_{c} \sum_{i=0}^{N-1} z_{l, i} x_{l, i}=T_{c} \operatorname{Cs}\left(z_{l}, x_{l}\right)
$$

where $\operatorname{Cs}\left(z_{l}, x_{l}\right)$ is the decision variable and is the discrete covariance sum from $\left(z_{l}, x_{l}\right)$. To demodulate the received signal the correlator first calculates the covariance between $r_{l}(t)$ and $u_{l}(t)$ for each bit of duration $N T_{c}$. The transmitted bit $b_{l}$ is demodulated as $\hat{b}_{l}$ where in terms of the decision variable

$$
\hat{b}_{l}= \begin{cases}+1 & \text { if } \operatorname{Cs}\left(z_{l}, x_{l}\right) \geq 0 \\ -1 & \text { if } C s\left(z_{l}, x_{l}\right)<0 .\end{cases}
$$

Examination of the covariance sum $C s\left(z_{l}, x_{l}\right)$ shows that it can be split into the three expressions $\Omega_{l}, \Psi_{l}, \Theta_{l}$, as

$$
\operatorname{Cs}\left(z_{l}, x_{l}\right)=\Omega_{l}+\Psi_{l}+\Theta_{l}
$$

where

$$
\Omega_{l}=b_{l} x_{l}^{T} x_{l}, \Psi_{l}=\sum_{k=1 \neq l}^{L} b_{k} x_{k}^{T} x_{l}, \Theta_{l}=x_{l}^{T} \varepsilon .
$$

In (16), $\Omega_{l}$ is the information variable for the bit $b_{l}$ to be demodulated and its value also depends on the magnitudes in the chaotic spreading segment $x_{l}$ of the $l^{\text {th }}$ user; $\Psi_{l}$ is the interference variable due to the existence of other users and depends both on their bit values and the chaotic spreading segments of all users; $\Theta_{l}$ is the noise variable of the $l^{\text {th }}$ user and its value depends on the AWGN noise and chaotic spreading segment of the $l^{\text {th }}$ user.

For good performance of the communication system, the interference and noise variable $\Psi_{l}+\Theta_{l}$ must be considerably smaller than the message bit variable $\Omega_{l}$. With many users the effect of the $\Psi_{l}$ may be large in comparison $\Theta_{l}$.

Overall performance will be assessed through bit error rate (BER) on the basis of (13)(16); this is the main accepted performance measure for many types of communication system and has been the focus of attention in CSK systems. Its computation presents both theoretical and practical difficulties.

Four main approaches have evolved and will be treated in the rest of the paper. The first and poorest approximate approach uses a simple Gaussian approximation (SGA) for the distribution of the covariance sum $C s\left(z_{l}, x_{l}\right)$, the second and third involve a conditional Gaussian approximation (CGA) which can be very accurate, and the fourth is an exact Gaussian approach (EGA) which is insightful but presents computational challenges. All four approaches assume an AWGN channel.

\section{Theory for BER by Simple Gaussian Approximation}


The SGA approach has its roots in binary phase shift keying (BPSK), [21], in which the bit energy is constant, unlike CSK, and going back at least to [13] and earlier conference presentations. In [16] an SGA expression was derived specifically for the BER of singleuser CSK taking into account its non-constant bit energy; in [15] and also in related earlier work, [25], [26], many complex SGA expressions for the BERs of multi-user CSK were given. The aim here is to gain theoretical intuition from generalization and mathematical conciseness in the statistical aspects of SGA results, rather than to advocate their practical use.

The overall bit error rate (BER) requires the conditional error probabilities $P(\hat{b}= \pm 1 \mid b=\mp 1)$. These are equal by symmetry and thus the overall BER does not depend on the proportions of the transmitted \pm values, and so

$$
B E R=P\left\{C s\left(z_{l}, x_{l}\right)<0 \mid b_{l}=1\right\} .
$$

The SGA approximation to the BER probability in (17) is based on the assumption that $C s\left(z_{l}, x_{l}\right)$ has a Gaussian distribution over the chaotic spreading, and so is

$$
Q\{E(C s) / \sqrt{\operatorname{var}(C s)}\}, Q(x)=\int_{x}^{+\infty} \frac{1}{\sqrt{2 \pi}} \exp \left(-u^{2} / 2\right) d u
$$

where $E(C s)$ and $\operatorname{var}(C s)$ are the mean and variance of $C s\left(z_{l}, x_{l}\right)$. There is no justification for the Gaussian distribution of $C s$, except for its summation structure. From (15)

$$
E\left\{C s\left(z_{l}, x_{l}\right)\right\}=E\left(\Omega_{l}\right)+E\left(\Psi_{l}\right)+E\left(\Theta_{l}\right)
$$

where the second and third expectations are zero and thus

$$
E\left\{C s\left(z_{l}, x_{l}\right)\right\}=N \sigma_{X}^{2} .
$$

The variance expression for $C s\left(z_{l}, x_{l}\right)$ is more complicated but there are no covariances between $\Omega_{l}, \Psi_{l}, \Theta_{l}$, and so only the sum of their variances is required. Individually, these require autocovariance terms within each of the chaotic spreading sequences. The final result, informative in regard to its constituent terms, is as follows

$\operatorname{Var}\left\{\operatorname{Cs}\left(z_{l}, x_{l}\right)\right\}=$

$$
N \sigma_{X}^{2}\left\{\left[(L-1) \sigma_{X}^{2}+\sigma_{n}^{2}\right]+\frac{\sigma_{X^{2}}^{2}}{\sigma_{X}^{2}}\left[1+2 \sum_{k=1}^{N-1}\left(1-\frac{k}{N}\right) \rho_{X^{2}}(k)\right]+2(L-1) \sigma_{X}^{2} \sum_{k=1}^{N-1}\left(1-\frac{k}{N}\right) \rho_{X}(k)^{2}\right\} \text {. }
$$

Here $\sigma_{X^{2}}^{2}$ denotes the variance of $X^{2}$, and $\rho_{X}(k), \rho_{X^{2}}(k)$ are the autocorrelations of $X$ and $X^{2}$, respectively.

The SGA method for calculating BER is given by applying (18) with (20) and (21) to yield

$B E R \square Q\left\{\left[\frac{1}{S N R}+\frac{1}{S O R}+N^{-1} \frac{\sigma_{X^{2}}^{2}}{\sigma_{X}^{4}}\left[1+2 \sum_{k=1}^{N-1}\left(1-\frac{k}{N}\right) \rho_{X^{2}}(k)\right]+\right.\right.$ 


$$
\left.\left.\frac{2}{S O R} \sum_{k=1}^{N-1}\left(1-\frac{k}{N}\right)\left\{\rho_{X}(k)\right\}^{2}\right]^{-\frac{1}{2}}\right\} .
$$

The notation $S N R=N \sigma_{X}^{2} / \sigma_{n}^{2}$, equivalently $E_{b} / N_{0}$, has been used to define the signalto-noise-ratio of the system and SOR defines the quantity $N /(L-1)$ which will be called the spreading-to-other-user-interference ratio. The result (22) is a more general and revealing form than found in earlier use. The first and the third terms refer to the active $l^{\text {th }}$ user while the second and fourth terms are due to the interfering other users. The autocorrelations suggest there is advantage in negative quadratic dependency and zero linear autocorrelations. The ratio term is actually the kurtosis of the spreading distribution. Each of these properties of the spreading may be obtained explicitly for some chaotic maps and by simulation for others. The accuracy of (22) can, however, be poor, as evident from the single-user case where it is evident that the BER does not even tend to one-half as the spreading extend tends to infinity. This comment is supported by Fig. 3 and Fig. 4.

It is of interest to compare the SGA method (22) with the constant bit energy case of BPSK, equivalently, antipodal DS-CDMA, for which the BER, from [21] for instance, is

$$
B E R_{B P S K}=Q\left\{\left(S N R^{-1}\right)^{-\frac{1}{2}}\right\} .
$$

This result is evident in the first term of (22) applied to the single-user case, emphasising that it is the simplest of approximations. Actually, as shown in [17], (23) is the lower bound for single-user CSK communication systems.

\section{Theory for BER by Conditional Gaussian Approximation}

Since the SGA method is very crude in its Gaussian assumption, a possibly better approach is to apply the Gaussian approximation conditionally on the chaotic spreading samples of the active user, and subsequently average over all chaotic spreading samples which the active user might employ. Thus $E(C s)$ and $\operatorname{var}(C s)$ are now considered conditionally on $x_{l}$ and denoted by $E\left(C s \mid x_{l}\right)$ and $\operatorname{var}\left(C s \mid x_{l}\right)$. Following on from (18), the CGA to the BER probability thus becomes

$$
E_{x_{j}}\left[Q\left\{E\left(C s \mid x_{l}\right) / \sqrt{\operatorname{var}\left(C s \mid x_{l}\right)}\right\}\right] .
$$

The required calculations of the conditional quantities yield the results

$$
\begin{aligned}
E\left(C s \mid x_{l}\right) & =\sum_{i=0}^{N-1} x_{l, i}^{2} \\
\operatorname{var}\left(C s \mid x_{l}\right) & = \\
& {\left[(L-1) \sigma_{X}^{2}+\sigma_{n}^{2}\right] \sum_{i=0}^{N-1} x_{l, i}^{2}++2 N(L-1) \sigma_{X}^{2} \sum_{k=1}^{N-1}\left(N^{-1} \sum_{i=0}^{N-k-1} x_{l, i} x_{l, i+k}\right) \rho_{X}(k) . }
\end{aligned}
$$

Hence, using (24), the CGA result for BER becomes 


$$
E_{x_{l}} \times Q\left[\left(\sum_{i=0}^{N-1} x_{l, i}^{2} / N \sigma_{X}^{2}\right)^{\frac{1}{2}}\left(\frac{1}{S N R}+\frac{1}{S O R}+\frac{2}{S O R} \sum_{k=1}^{N-1}\left\{\sum_{i=0}^{N-k-1} x_{l, i} x_{l, i+k} / \sum_{i=0}^{N-1} x_{l, i}^{2}\right\} \rho_{X}(k)\right)^{-\frac{1}{2}}\right]
$$

where the sum of squares term is the standardized chaotic bit energy for the typical $j^{\text {th }}$ bit. The expression (27) facilitates CGA comparison with the SGA formula (22); for instance, the empirical and theoretical autocorrelations align with the squared autocorrelations. With uncorrelated spreading there is the much simpler result without the autocorrelations. With extensive enough spreading, the empirical autocorrelations can be replaced by the corresponding theoretical ones, to yield the simpler general result

$$
B E R \square E_{x_{l}} Q\left[\left(\sum_{i=0}^{N-1} x_{l, i}^{2} / N \sigma_{X}^{2}\right)^{\frac{1}{2}}\left(\frac{1}{S N R}+\frac{1}{S O R}+\frac{2}{S O R} \sum_{k=1}^{N-1}\left(1-\frac{k}{N}\right)\left\{\rho_{X}(k)\right\}^{2}\right)^{-\frac{1}{2}}\right]
$$

Ignoring the terms involving SOR, the single-user exact result [17] is seen here.

\section{Chaotic Maps in CSK}

In order to better understand the effect of chaotic maps $\tau(x)$ on the distribution of bit energy, three cases have been chosen, two giving linearly uncorrelated spreading and one giving both linearly and quadraticly autocorrelated spreading. The first linearly uncorrelated one is the well known logistic map with

$$
\tau(x)=2 x^{2}-1, \quad-1<x<1,
$$

and which has a particular beta invariant distribution. The second is the PWL map, sonamed in [3] and illustrated in Figure 2. Its defining equation is

$$
\tau(x)=\operatorname{sign}(x)\{([2(K|x|+\varphi)])(\bmod 1)-1\},-1<x<1 .
$$

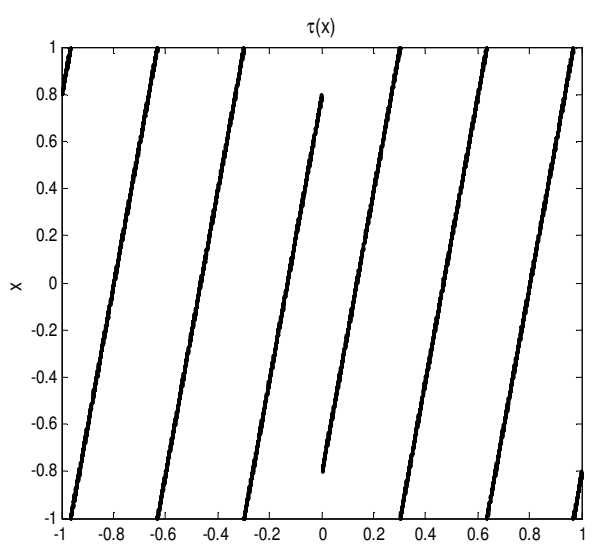

Fig. 2 . The PWL map with $K=3, \phi=0.1$.

This is a generalized shift map with $2 K+2$ branches of slope $2 K$; the first, last and middle two of which are not complete and so represent deviations from a standard shift 
map; the parameter $\varphi(0<\varphi<1)$ controls the incomplete branches. In [3] it is shown to have a uniform invariant distribution; it can be both linearly and quadraticly autocorrelated, but in the case used throughout this paper, $K=3, \varphi=0.1$, these autocorrelations are very small or effectively zero. The third map to be used is the well known Bernoulli-shift map,

$$
\tau(x)=\left\{\begin{array}{cc}
2 x+1 & -1<x \leq 1 \\
2 x-1 & 0<x<1
\end{array}\right.
$$

which has a uniform invariant distribution and is geometrically linearly and quadraticly autocorrelated with parameters one-half and one-quarter, respectively. The three contrasting maps (29), (30) and (31) are each used to generate one million chaotic segments of 10

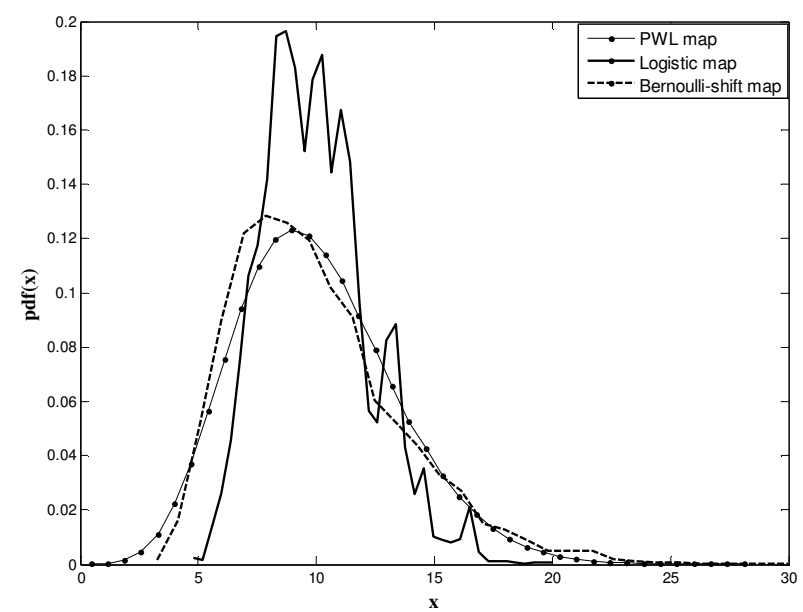

Fig. 3: Simulated distributions of bit energy for a spreading factor of 10 from chaotic sequences generated by logistic, PWL and Bernoulli-shift maps.

samples, that is a spreading factor of 10. Fig. 3 shows the simulated distributions of bit energy where it can be seen that the logistic one is the narrowest. Thus, the logistic map will give the best results in terms of BER, although its multimodal shape can be a disadvantage in subsequent BER calculation. This shape appears to be a consequence of the beta invariant distribution of the logistic map being $\mathrm{u}$-shaped and thus having most of its values towards the \pm 1 boundaries.

Although the logistic map is the best of those mentioned, theoretically optimal spreading in which the variability of bit energy is minimised has been developed in [29], [18] and leads to so-called circular and deformed-circular maps; this topic will not be expounded here.

\section{BER by CGA and Simulation of Bit Energy}

In this section the use of the CGA result (28) involving the distribution of bit energy in calculating BER is developed. It can be applied whether the chaotic samples are linearly uncorrelated or not. Two approaches to the distribution of bit energy are considered. Both require the simulation of the bit energy distribution, the first (CGA-Simulation) employs the simulated histogram directly in a numerical integration, while the second (CGA- 
Analytical) fits a particular probability density to the histogram and uses this in an analytical integration. These two methods are explained in this and the following Section 7. The methods lead to a highly accurate performance prediction with low computing charge, as evident in [8] for uncorrelated spreading, and here for autocorrelated spreading as well.

Now, according to the CGA result (28), the BER result can be written as the integral

$$
B E R_{c s k}=\int_{0}^{+\infty} Q\left\{\sqrt{e_{b c} /\left(\frac{1}{S N R}+\frac{1}{S O R}+\frac{2}{S O R} \sum_{k=1}^{N-1}\left(1-\frac{k}{N}\right)\left\{\rho_{X}(k)\right\}^{2}\right)}\right\} f\left(e_{b c}\right) d e_{b c}
$$

where $e_{b c}$ is the standardized chaotic bit energy variable for the typical $j^{\text {th }}$ bit and $f\left(e_{b c}\right)$ is its probability density function. However, an analytical expression for $f\left(e_{b c}\right)$ is difficult because the chaotic samples are not statistically independent, rather they are functionally dependent. One easier solution is to simulate bit energy and so obtain an accurate probability density function representation as in Fig. 3 and then numerically integrate (32). A comment based on (32) is that BER is not zero even for a noise-free channel (infinite SNR) because of the interference effect of other-users represented by SOR terms.

\section{The CGA-Simulation Method}

The CGA-simulation method takes the formula (32) and converts the analytical integration to numerical integration as

$$
B E R_{c s k} \square \sum_{j=1}^{m} Q\left\{\sqrt{E_{b c}^{(j)} /\left(\left(\frac{1}{S N R}+\frac{1}{S O R}+\frac{2}{S O R} \sum_{k=1}^{N-1}\left(1-\frac{k}{N}\right)\left\{\rho_{X}(k)\right\}^{2}\right)\right)}\right\} P\left(E_{b c}^{(j)}\right)
$$

where $m$ is the number of histogram classes created from the simulated spreading segments and $P\left(E_{b c}^{(j)}\right)$ is the fraction of the 1 million segments having the energy in the interval centered on $E_{b c}^{(j)}$.

The strength of the CGA-Simulation method is that it can be applied very simply for any type of uncorrelated chaotic sequence, needing only a simulated distribution of the bit energy and an easy subsequent a numerical integration. With autocorrelated spreading, additionally, the sums of squares of autocorrelations are required but can be obtained from known analytical results or simulation. Extensive and fast computing enables these simulation results to be obtained with high accuracy, which then allow accurate numerical integration, even in multi-modal cases. Moreover, due to its low computational charge, the method can be implemented for realistic sized systems. A theoretical strength is that by invoking chaotic map simulations, the chaotic dynamics of the map are properly utilized and allow comparisons between different types of chaotic spreading. 


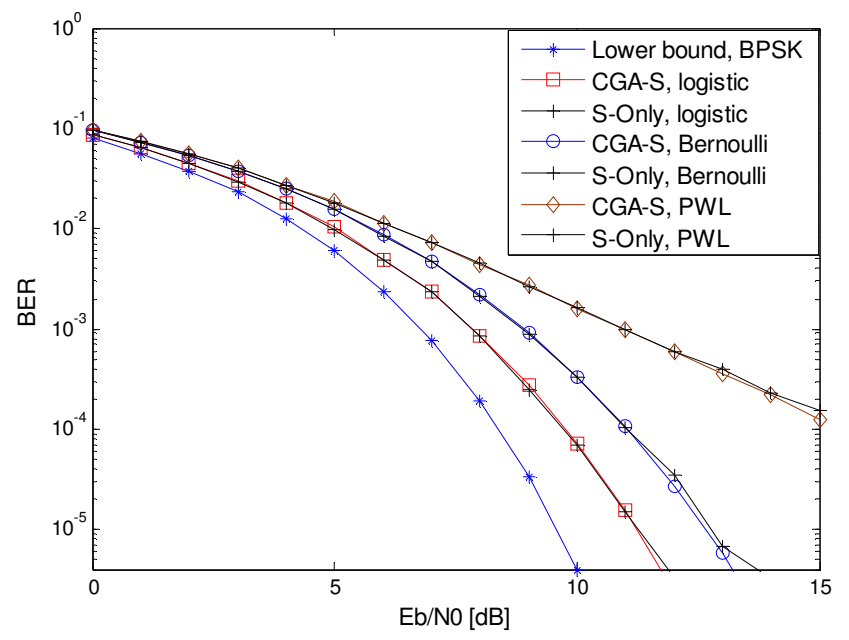

Fig. 4. BER for single-user CSK with logistic, Bernoulli-shift and PWL spreading, $N=5$. CGA-Simulation (CGA-S) and Simulation-Only (S-Only) methods. Also shown is the BPSK lower bound curve.

For single-user CSK, in which the terms involving SOR in (33) are omitted, Fig. 4 gives simulation-only BER results, together with the CGA-Simulation method of calculation. BER spreading is by the uncorrelated logistic and PWL maps and the autocorrelated Bernoulli-shift map, each with spreading factor $N=5$. Also shown is the lower bound corresponding to the BPSK system. The near-perfect match between the simulation-only results and the CGA-Simulation method confirms the accuracy of this approach. It can be seen that logistic spreading is superior to PWL and Bernoulli-shift spreading, but that the spreading factor of $N=5$ is not large enough to give BER performance close to that of BPSK. The larger the spreading factor, the less variable is the standardized bit energy and so closer to the constant bit energy of BPSK, as previously illustrated in Fig. 3.

Fig. 5 gives a comparison of the SGA method (22) and the CGA-Simulation method (33) in calculating BER for single-user CSK with the uncorrelated logistic spreading, $N=5$ and $N=100$. The upper-most curve gives the inaccurate SGA results. The lowest group of curves consists of the BPSK lower bound, the CGA-Simulation curve and the Simulation-only curve; the closeness of these illustrates the excellent accuracy of CGASimulation for high spreading. The middle group of two curves compare the CGASimulation results with those of the Simulation-only values. The close agreement here attests to the high accuracy of the CGA-simulation method for spreading as low as $N=5$. 


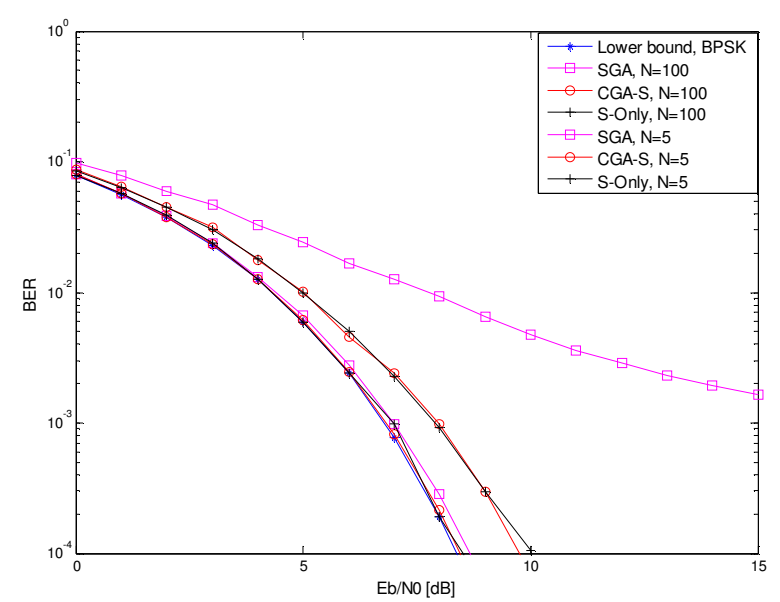

Fig. 5. BER for single-user CSK with logistic spreading, $N=5$,100. SGA, CGA-Simulation (CGA-S) and Simulation-Only (S-Only) methods. Also shown is the BPSK lower bound curve.

Continuing to multi-user CSK and the further use of (22) and (33), Fig. 6 illustrates the high accuracy of the CGA-Simulation method for the uncorrelated logistic spreading with $N=10$ and $L=3$ users, and for $L=2$ users with the uncorrelated PWL spreading, $N=30$. Fig. 7 is concerned with the autocorrelated Bernoulli-shift spreading and with the need for autocorrelation in the CGA-Simulation formula (33). The lower group of three curves refers to $(L=2, N=20)$ while the upper group refers to $(L=2, N=10)$. In each group, there is a separation of the curve corresponding to the omission of autocorrelation in (33) from the CGA-Simulation and Simulation-only curves. This is convincing evidence of autocorrelation terms being needed in (33) for accurate results. Figure 8 continues the illustrations in Fig. 7 with Bernoulli-shift spreading to the higher number of

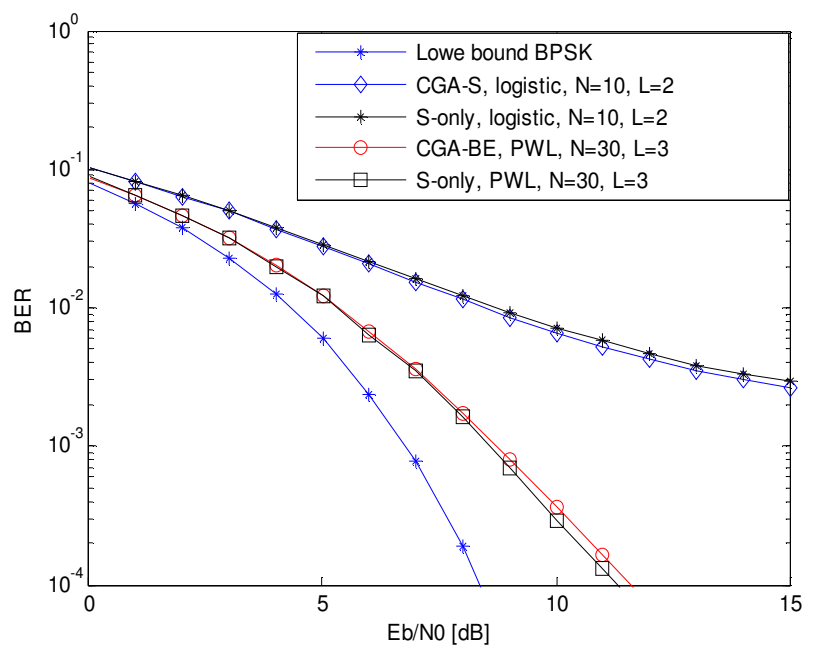

Fig. 6. BER for multi-user ( $L=2,3)$ CSK with logistic and PWL spreading, $N=10,30$. CGA-Simulation (CGA-S) and Simulation-Only (S-Only) methods. Also shown is the BPSK lower bound curve. 


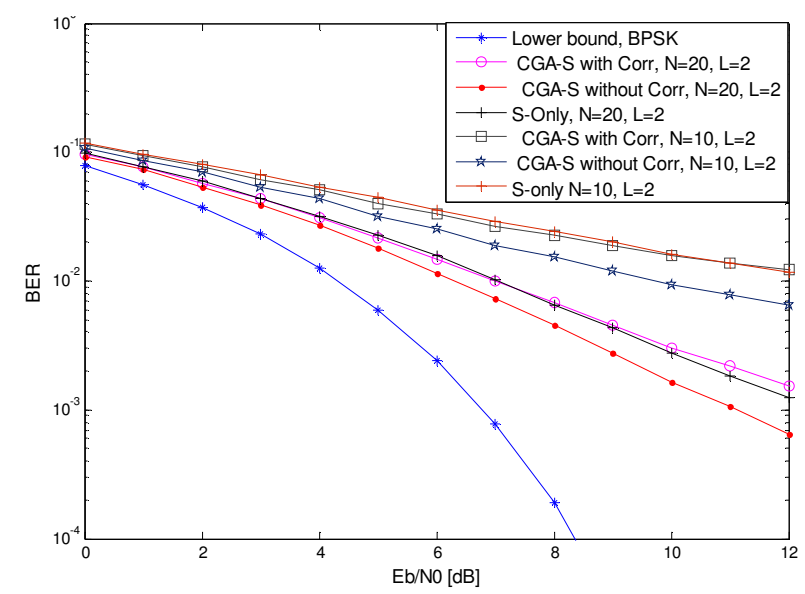

Fig. 7. BER for multi-user $(L=2)$ CSK with Bernoulli-shift spreading, $N=10,20$. CGA-Simulation (CGA-S) with and without autocorrelation (with Corr, without Corr) and Simulation-Only (S-Only) methods. Also shown is the BPSK lower bound curve.

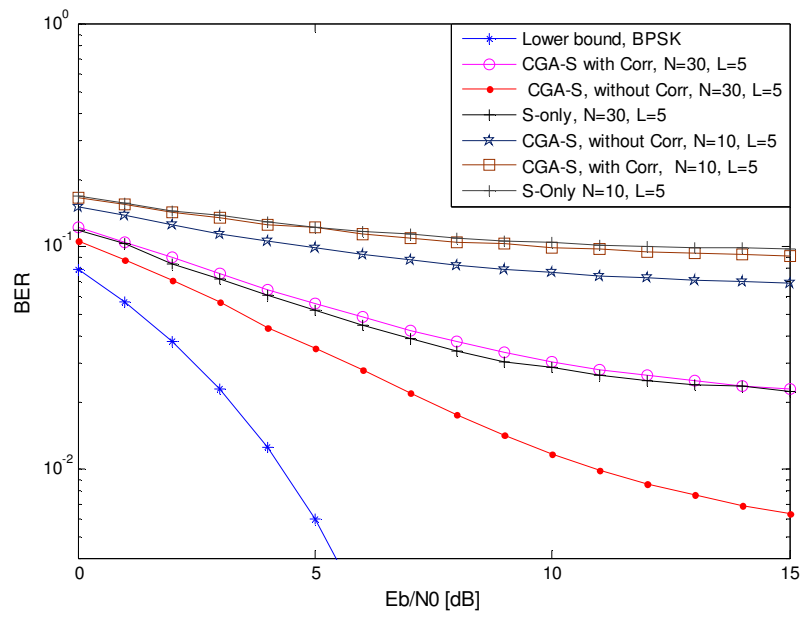

Fig 8. BER for multi-user $(L=5)$ CSK with Bernoulli-shift spreading, $N=10,30$. CGA-Simulation Energy (CGA-S) with and without autocorrelation (with Corr, without Corr) and Simulation-Only (S-Only) methods. Also shown is BPSK lower bound curve.

4

$L=5$ users and spreading $N=10, N=30$. There is the same pattern of results, that with the higher number of users CGA-Simulation is still accurate, and with an accentuation of the need for autocorrelation in (33) at high spreading.

\section{BER by CGA and Analytical Distribution of Bit Energy}

The CGA-Analytical method for computing BER, more mathematical than the CGA simulation method, is based on detailed analytical results for the distribution of bit energy which have been obtained previously in the context of mobile radio channels, [1], [20], [7] and [5]. This method uses (32) in terms of the square root of bit energy (SRE), $\lambda=\sqrt{e_{b c}}$, and the expression

$$
B E R_{c s k} \square \int_{0}^{+\infty} Q\left(\sqrt{\lambda^{2} /\left(\frac{1}{S N R}+\frac{1}{S O R}+\frac{2}{S O R} \sum_{k=1}^{N-1}\left(1-\frac{k}{N}\right)\left\{\rho_{X}(k)\right\}^{2}\right)}\right) p(\lambda) d \lambda .
$$


In mobile radio channel work, the square root of bit energy, as channel attenuation, follows either the Rayleigh distribution, [5], the Nakagami distribution, [7] or the Rice distribution [20], and in these cases analytical expressions are available for (34). Thus, the immediate task is to ascertain whether the simulated pdf of the square root of bit energy for CSK systems can be satisfactorily matched to one of these distributions. In this way, a fully analytical expression, although approximate to some limited degree, will be obtained.

Recall from Fig. 3 that the logistic spreading segments have given an irregular shape to the bit energy distribution and thus not analytically suitable to probability density function matching. On the other hand, the PWL simulated distribution appears smooth and unimodal, although skewed. Thus, continuing with $10^{6}$ PWL segments with spreading factor $N=10$, the SRE simulated distribution has been obtained and is shown in Fig. 9; also shown are the probability density functions of the Rice and Nakagami distributions. Both are visually very close to the PWL simulated distribution, but according to the chi-square goodness-of-fit test, the Rice distribution is to be preferred. The Rice distribution function is given by (53) in Appendix A.

\section{The CGA Analytical Method using the Rice Distribution and PWL spreading}

In order to obtain the CGA analytical BER expression, the scale and shape parameters $\Omega, K$ of the Rice distribution must be computed in terms of the PWL map (30), as in [3]. This can be achieved by the assumption that the standardized bit energy from a PWL map is the square of a Rice variable $R$. The expectation of $R^{2}$ will then be unity, so requiring that the mean of the Rice distribution be unity. The chaotic values $x_{l, j}$ are uniformly distributed over the interval $[-1,+1],[3]$, and thus the variance of $R^{2}$, using the sum of variances formula, is

$$
\operatorname{var}\left(R^{2}\right)=\frac{4}{5 N}\left\{1+2 \sum_{k=1}^{N-1}\left(1-\frac{k}{N}\right) \operatorname{corr}\left(x_{l, i}^{2}, x_{l, i+k}^{2}\right)\right\}
$$

Here the quadratic autocorrelations have been ignored for the PWL as being very small in the case used. It is convenient to note from (53) and (54) in Appendix A for the Rice distribution that

$$
\Omega=E\left(R^{2}\right)=1, K_{r}=\frac{\sqrt{1-\gamma}}{1-\sqrt{1-\gamma}}, \gamma=\operatorname{Var}\left[R^{2}\right],
$$

and then use (55) and (56) in Appendix A for the final result. The comparisons in Fig. 9 indicate very accurate matching of both Rice and Nakagami distributions to the Simulation-only curve. 


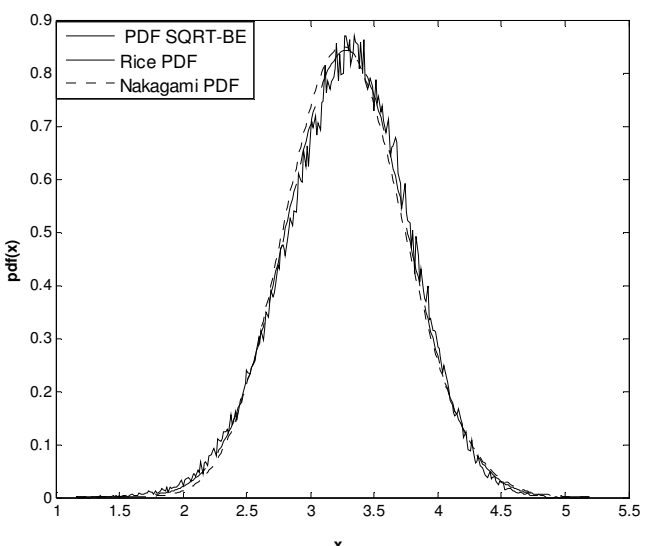

(a)

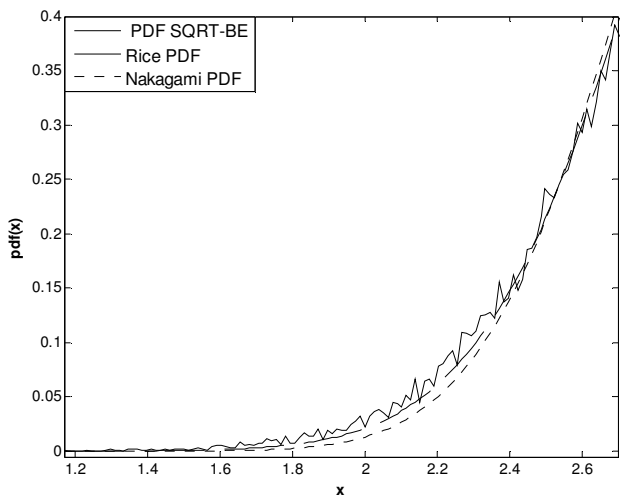

(b)

Fig. 9. (a) Comparison of the simulated distribution of the square root of bit energy for the PWL $\operatorname{map}(K=3, \varphi=0.1, N=10)$ with that of matching Rice and Nakagami probability density functions, (b)

Zoom in to show that the simulated distribution follows the Rice probability density function.

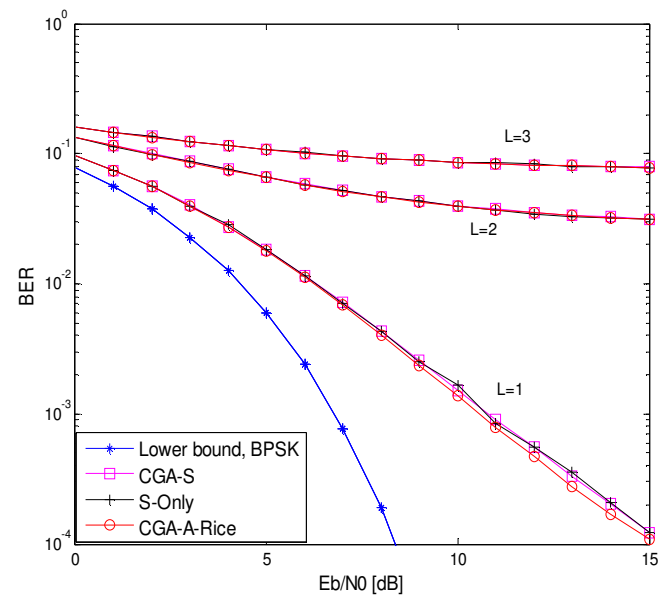

Fig 10. BER for multi-user ( $L=1,2,3) C S K$, with $P W L(K=3, \phi=0.1)$ spreading, $N=5$. CGA-Analytical Rice distribution (CGA-A-Rice), CGA-Simulation (CGA-S) and Simulation-Only (S-Only) methods. Also shown is the BPSK lower bound curve. 


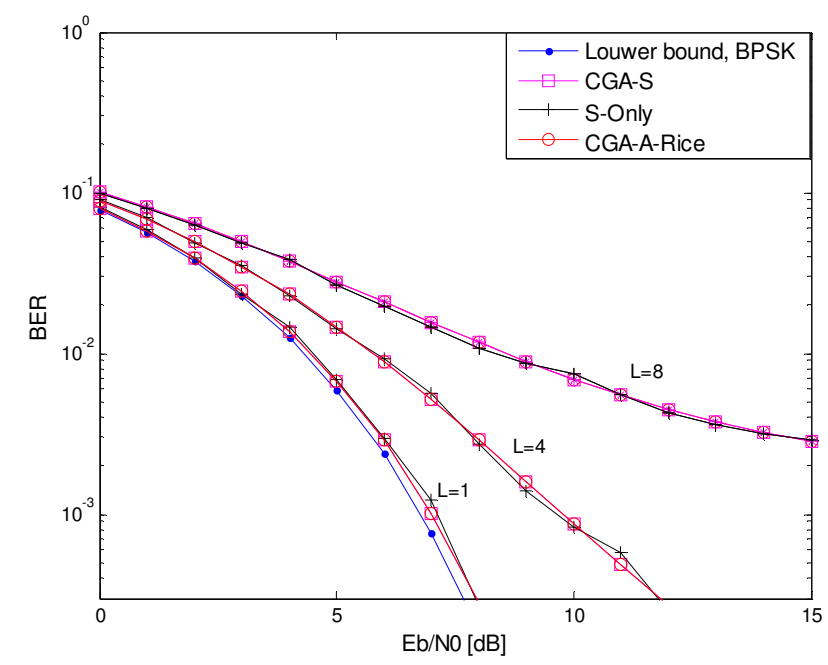

Fig. 11. BER for multi-user ( $L=1,4,8)$ CSK, with PWL $(K=3, \phi=0.1)$ spreading, $N=64$. CGA Analytical Rice distribution (CGA-A-Rice), CGA-Simulation (CGA-S) and Simulation-Only (S-Only) methods. Also shown is the BPSK lower bound curve.

The CGA analytical BER expression derived from (34) when the pdf of the square root energy is assumed to have a Rice distribution is, following [20],

$$
B E R_{P W L}=Q(u, v)-\frac{1}{2}\left[1+\sqrt{\frac{d}{1+d}}\right] \exp \left(-\frac{u^{2}+v^{2}}{2}\right) I_{0}(u v)
$$

where $Q(u, v), u, v, d$ are given in the Appendix B.

In Fig. 10 the CGA analytical BER expression from (37) is compared with results from Simulation-only (S-only) of CSK systems. For the low spreading factor of $N=5$ in these multi-user cases, there is excellent agreement, except at large signal-noise ratios; but then there are only small deviations. These results thus justify the approximations which have been made in obtaining the analytical result (37).

Fig. 11 gives similar results to Fig. 10 except for the larger spreading factor of $N=64$ and the larger numbers of users. The previous conclusions are reinforced. Furthermore, there is good agreement between the CGA-Analytical and the CGA-Simulation methods.

\section{Exact Gaussian Theory (EGT) for BER}

The Exact Gaussian Theory (EGT) for BER from correlation decoding in multi-user CSK is developed from the single-user case [17] and was initiated in [30]. First, by combining the covariance sum of the correlator (15) with its components (16) into a single expression, the BER probability in (17) becomes

$$
B E R=P\left\{C s\left(z_{l}, x_{l}\right)<0 \mid b_{l}=1\right\}=P\left(-\varepsilon_{l}^{T} x_{l}>x_{l}^{T} x_{l}+x_{l}^{T} \sum_{k=1 \neq l}^{L} b_{k} x_{k} \mid b_{l}=1\right) .
$$

Conditional on the spreading of the $l^{\text {th }}$ user, $\varepsilon_{l}^{T} x_{l}$ has a Gaussian distribution of mean zero and variance $\sigma_{n}^{2}\left(x_{l}^{T} x_{l}\right)$; thus conditional on spreading and bit values of the other users 
as well, the BER probability can be written

$$
Q\left(\left\{x_{l}^{T} x_{l}+\Psi_{l}\right\} / \sigma_{n}\left(x_{l}^{T} x_{l}\right)^{\frac{1}{2}}\right), \Psi_{l}=x_{l}^{T} \sum_{k=1 \neq l}^{L} b_{k} x_{k} .
$$

This means that (39) can be regarded as a function two dependent random variables, bit energy $x_{l}^{T} x_{l}$ and the multi-user variable $\Psi_{l}$, also from (16). The unconditional BER is thus the average of $Q$ over the bit energy and the multi-user variable. The single-user case will be presented first.

\section{Single-User Exact Gaussian Theory}

Exact theory for the single user case of CSK begins from (39) without the $\Psi_{l}$ term. Hence, and after a little rearrangement, the BER expression is

$$
B E R=E_{x_{l}} Q\left\{\left[\left(N^{-1} x_{l}^{T} x_{l} / \sigma_{X}^{2}\right) S N R\right]^{1 / 2}\right\}
$$

in terms of standardized bit energy, the result from [17]. For exact results, the expectation has to be evaluated numerically. This might seem to need and $\mathrm{N}$-dimensional integral, but in the chaotic case is one-dimensional because

$$
x_{l, i}=\tau^{(i-1)}\left(x_{0}\right), \quad i=0,1,2, \ldots
$$

where $\tau^{(i)}(\cdot)$ is the $i^{\text {th }}$ iteration of the map, and the expectation can be done as a function of the single variable $x_{0}$ which has the invariant distribution of the map, pdf $f_{X}(\cdot)$. More explicitly, it can be seen that (40) leads to

$$
B E R=\int_{-1}^{+1} \Phi\left\{-\sqrt{S N R}\left(N^{-1} \sum_{j=1}^{N} \tau^{(j-1)}(x)^{2} / \sigma_{X}^{2}\right)^{1 / 2}\right\} f_{X}(x) d x .
$$

Explicit calculations require mathematical formulae for the map iterations, as are available for standard maps, but not for many others. Otherwise, these have to be done numerically, a process which is bound to degrade accuracy. For logistic and Bernoulli-shift maps, as specified at (29) and (31), for $j=2,3, \ldots$, there are

$$
\begin{gathered}
\tau^{(j)}(x)=\cosh \left(2^{j-1} \operatorname{arccosh}(x)\right),-1 \leq x<1, \\
\tau^{(j)}(x)=2^{j-1} x-1-2 k, \quad \frac{k}{2^{j-2}} \leq x<\frac{k+1}{2^{j-2}}, k=-2^{j-2}, \ldots, 2^{j-2}-1 .
\end{gathered}
$$

These functions allow (42) to be coded in any desired language, with computational accuracy governed only by software; they also allow the exact distribution of standardized bit energy to be obtained as a transformation of the invariant distribution. Illustrations of exact BER for logistic and Bernoulli-shift maps are given in Fig.12. They show that logistic spreading is superior to Bernoulli-shift spreading, a result might have been anticipated. 


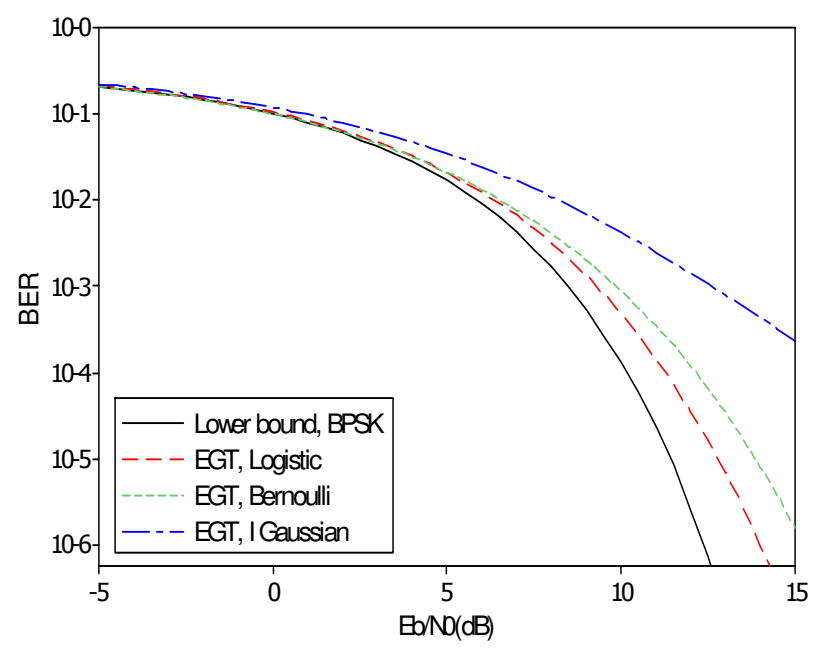

Fig. 12. BER for single-user CSK by exact Gaussian theory (EGT) method with logistic, Bernoulli-shift and independent Gaussian spreading, N=5. Also shown is the BPSK lower bound curve.

from the SGA result (22) because logistic map spreading is not linearly autocorrelated, unlike Bernoulli-shift map. However, the mathematical exactness of these results is their value. Note that non-chaotic independent Gaussian spreading is the least effective, although it is still much lower than the $N=1$ upper bound which is not shown.

One insightful deduction from the exact result (40) is that BER has $Q(\sqrt{S N R})$ as its lower bound, as already noted at (23), and further that this arises when bit energy is constant. An upper bound is the $N=1$ case. Another deduction from (40) is concerned with minimising bit energy for optimum spreading, and shows that the spreading should have negative quadratic autocorrelations; this conclusion is foreshadowed in the SGA result (22) and first reported in [29].

\section{Multi-user Exact Gaussian Theory}

Exact Gaussian theory for BER from correlation decoding in multi-user CSK begins from the expectation of (39) over all spreading $x_{1}, \ldots, x_{L}$ and over the bit values of all other users, $b_{1}, \ldots<b_{l}>\ldots, b_{L}$. Denoting these more briefly as $x, b$, there is the following most general result

$$
B E R=E_{x, b} Q\left(\left\{x_{l}^{T} x_{l}+\Psi_{l}\right\} / \sigma_{n}\left(x_{l}^{T} x_{l}\right)^{\frac{1}{2}}\right) .
$$

To take this further in full exactness is a combinatorial calculation which involves assuming that the proportions of the transmitted \pm 1 bit values are $p: 1-p$ and that $r$ of the other users transmit +1 's $, r=1,2, \ldots, L-1$. Then there is the 'perfectly exact' expression

$$
B E R=\sum_{r=1}^{L-1}\left(\begin{array}{c}
L-1-r \\
r
\end{array}\right) p^{r}(1-p)^{L-1-r} E_{x} Q\left(\left\{x_{l}^{T} x_{l}+\sum_{k=1 \neq l}^{r} x_{k}^{T} x_{l}-\sum_{k=r+1 \neq l}^{L-1} x_{k}^{T} x_{l}\right\} / \sigma_{n}\left(x_{l}^{T} x_{l}\right)^{\frac{1}{2}}\right) .
$$

This result is computationally rather difficult, but a more tractable result is based on (39) 
and the use of conditional expectations. As a function of its two variables, $x_{l}$ and $\Psi_{l}$, (39) gives for BER the expression

$$
B E R=E_{x_{l}} E_{\Psi_{l} \mid x_{l}} Q\left(\left\{x_{l}^{T} x_{l}+\Psi_{l}\right\} / \sigma_{n}\left(x_{l}^{T} x_{l}\right)^{\frac{1}{2}}\right) .
$$

The conditional distribution of $\Psi_{l}$ clearly has a mean of zero, and a calculation for its variance, which recognizes the independence of spreading between different users, yields

$$
\operatorname{var}\left\{\Psi_{l}\right\}=(L-1)\left(x_{l}^{T} \Sigma_{X} x_{l}\right) \sigma_{X}^{2}
$$

where $\Sigma_{X}$ is the $N \times N$ circulant autocorrelation matrix of the spreading. Particularly note that the variance does not involve the bits of other users since the calculation has involved their squaring, a great saving in complication. The next step is one of accurate approximation for the conditional distribution of given $x_{l}$, and since $\Psi_{l}$ is the sum of $L-1$ independent terms, this can be taken as Gaussian. The resulting calculation in (47) is done by using the formula (60) in Appendix C, giving the nearly EGT result

$$
B E R_{l}=E_{x_{l}}\left\{\Phi\left(-x_{l}^{T} x_{l}\left[\sigma_{\varepsilon}^{2} x_{l}^{T} x_{l}+(L-1) \sigma_{X}^{2} x_{l}^{T} \Sigma_{X} x_{l}\right]^{-1 / 2}\right)\right\} .
$$

This can be rearranged as follows

$$
B E R=E_{x_{l}}\left\{Q\left(\left(N^{-1}\left(x_{l}^{T} x_{l}\right) / \sigma_{X}^{2}\right)^{1 / 2}\left(\frac{\left.x_{l}^{T} x_{l}\right)}{\left.x_{l}^{T} \Sigma_{X} x_{l}\right)}\right)^{1 / 2}\left[\left(\frac{\left.x_{l}^{T} x_{l}\right)}{\left.x_{l}^{T} \Sigma_{X} x_{l}\right)}\right) \frac{1}{S N R}+\frac{1}{S O R}\right]^{-1 / 2}\right)\right\}
$$

which is reminiscent of the earlier more approximate SGA and CGA results (22) and (27). With uncorrelated spreading it reduces to the CGA result (32) but not so with autocorrelated spreading. With no channel noise, there is simplification which indicates there is still bit error caused by the interference of other users. This is the explanation of the eventual horizontal behaviour of multi-user BER curves beginning to be evident in Fig. 6 - Fig. 8 , Fig. 10 - Fig.12, and Fig. 13 includes an illustration of the near exact result (50) for Bernoulli-shift map spreading.

\section{Generalized Correlation Decoding}

Up to this point the paper has been concerned with correlation decoding, the standard method. However, a decoder is only optimal with minimum BER when it is the maximum likelihood estimator of the transmitted bit. The correlation decoder is only the maximum likelihood estimator for Gaussian channel single-user CSK systems. There has been some previous investigation of likelihood decoding, e.g. [22], but not for multiple-user CSK in the coherent form. 


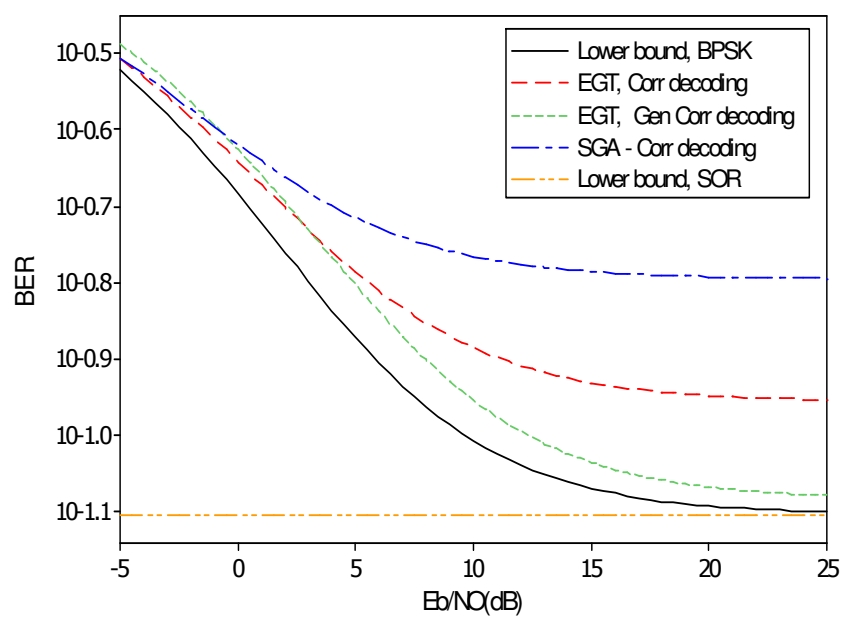

Fig. 13. BER for multi-user $L=3$ CSK with Bernoulli-shift spreading, $N=4, S O R=2$. SGA for correlation decoding, EGT for correlation decoding, and EGT for generalized correlation decoding. Also shown are the BPSK lower bound for uncorrelated spreading and the noiseless SOR lower bound.

In general it is a formidable problem and no explicit decoder is derivable, and therefore its analytical BER cannot be envisioned. However, in [19] the likelihood was approximated by a multivariate Gaussian distribution and this led to a generalized correlation decoder which took the form

$$
\hat{b}_{l}= \begin{cases}+1 & \text { if } z_{l}^{T} \Sigma_{X}^{-1} x_{l} \geq 0 \\ -1 & \text { if } z_{l}^{T} \Sigma_{X}^{-1} x_{l}<0\end{cases}
$$

where $\Sigma_{X}^{-1}$ is the inverse of the circulant autocorrelation matrix of the spreading vector $x_{l}$. This decoder is thus seen to take account of autocorrelation within the spreading, and to be the same as the correlation decoder if there is no autocorrelation within the spreading. The exact theory for its BER follows similarly to that of the correlation decoder at (38) and (39), and the result from [19] is

$$
B E R=E_{x_{l}}\left\{Q\left(\left(N^{-1} x_{l}^{T} \Sigma_{X}^{-1} x_{l} / \sigma_{X}^{2}\right)^{1 / 2}\left[\left(\frac{x_{l}^{T} \Sigma_{X}^{-1} \Sigma_{X}^{-1} x_{l}}{x_{l}^{T} \Sigma_{X}^{-1} x_{l}}\right) \frac{1}{S N R}+\frac{1}{S O R}\right]^{-1 / 2}\right)\right\} .
$$

In the case of three users and Bernoulli-shift map spreading of factor 4, exact calculations from (52) are used in Fig. 13 to compare the BER with the corresponding result (50) for the correlation decoder. The comparison illustrates in a very simple case the advantage of the generalized correlation decoder over the conventional one, from taking advantage of the autocorrelation in the Bernoulli-shift map spreading. Evidence here seems to suggest that for high SNR, the generalized correlation decoder has considerably lower BER than the correlation decoder but for negative or modest SNR it could be a negligible amount greater. Also in Fig. 13 are the inaccurate SGA (22) for correlation decoding, the BPSK lower bound (23) and the noiseless SOR lower bound $Q(\sqrt{S O R})=0.07865$. 


\section{Conclusions}

The purpose of the paper has been to examine, extend and inter-relate the theory and methods of calculation of bit error rate for single and multi-user coherent chaos shifting systems, using correlation decoders and a likelihood-based improvement. The SGA method has been viewed as valuable in concisely indicating the key statistical quantities which effect BER but not for yielding generally accurate results. A more accurate CGA method is developed and its results are compared to their SGA counterparts; these involve the use of the distribution of bit energy and quadratic autocorrelations, unusual aspects relative to conventional communication systems. Consideration has also been given to exact calculation of BER, the 'gold standard', usually insightful but computationally much more demanding. Finally, theory has also been presented for maximum likelihoodbased generalized correlation decoders and their improved BER performance. The relative advantages and disadvantages of the different approaches have been illustrated. Similar analyses can be applied to non-coherent CSK and other chaotic systems.

\section{Acknowledgements}

Dr G Kaddoum acknowledges support by the EPSRC Centre for Research in Statistical Methodology (CRiSM) in the Department of Statistics at the University of Warwick during a visit in May 2009 to work on this paper.

\section{APPENDICES}

\section{Appendix A. The Rice distribution}

A random variable $R$ with a Rice distribution has distribution function, [20], given by

$$
p(r)=\frac{2(K+1) r}{\Omega} \exp \left(-K-\frac{(K+1) r^{2}}{\Omega}\right) I_{0}\left(2 \sqrt{\frac{K(K+1) r}{\Omega}}\right), r \geq 0
$$

where $\Omega \geq 0, K \geq 0$ are the scale and shape parameters, and $I_{0}(x)$ is the zero-order modified Bessel function of the first kind. The scale and shape parameters $\Omega$ and the $K$ are given, simplified from [1], in terms of the standardized bit energy moments by

$$
\Omega=E\left(R^{2}\right), K_{r}=\frac{\sqrt{1-\gamma}}{1-\sqrt{1-\gamma}},
$$

where $\gamma=\operatorname{Var}\left[R^{2}\right] /\left(E\left(R^{2}\right)\right)^{2}$ is the kurtosis of $R$. Defining the following further parameters as

$$
\sigma^{2}=\frac{\Omega}{2(K+1)}, \alpha^{2}=\frac{K \Omega}{(K+1)},
$$

a convenient form of the Rice pdf is then given, as in [1], by 


$$
p_{R}(r)=\frac{r}{\sigma^{2}} \exp \left(-\frac{r^{2}+\alpha^{2}}{2 \sigma^{2}}\right) I_{0}\left(\frac{\alpha r}{\sigma^{2}}\right), r \geq 0
$$

and is used in the analytical formula (37) for BER.

\section{Appendix B. Rice distribution formulae for bit energy}

The following formulae have been needed to give the Rice approximation to BER with CSK pwl spreading,

$$
\begin{gathered}
v=\sqrt{\frac{\gamma^{2}[1+2 d+2 \sqrt{d(d+1)}]}{2(1+d)}}, u=\sqrt{\frac{\gamma^{2}[1+2 d-2 \sqrt{d(d+1)}]}{2(1+d)}} \\
\gamma^{2}=\frac{\alpha^{2}}{2 \sigma^{2}}, d=\sigma^{2} \frac{N \sigma_{X}^{2}}{\sigma_{n}^{2}},
\end{gathered}
$$

where $Q(\alpha, \beta)$ is the Marcum Q-function, [20], given by

$$
Q(\alpha, \beta)=\frac{1}{\alpha^{M-1}} \int_{x=\beta}^{\infty} x^{M} e^{-\left(x^{2}+\alpha^{2}\right) / 2} I_{M-1}(\alpha x) d x
$$

and $I_{M-1}(x)$ is a modified Bessel function of the first kind of order $M-1$.

\section{Appendix C. A Gaussian integral result}

With $\phi(\cdot)$ the probability density function of a standardized Gaussian variable, and for any constants $a, b, c, d$,

$$
\int_{-\infty}^{+\infty} b^{-1} \phi\left\{b^{-1}(m+a)\right\} Q\left\{-d^{-1}(m+c)\right\} d m=\Phi\left\{(c-a)\left(b^{2}+d^{2}\right)^{-1 / 2}\right\}
$$

\section{References}

[1] A. Abdi, C. Tepedelenlioglu, M.Kaveh, and G. Giannakis, "On estimation of K parameter for Rice fading distribution," IEEE Communications Letters, vol. 5, pp. 92-94, 2001.

[2] A. D. Argyris, D. Syvridis, L. Larger, V. Annovazzi-Lodi, P. Colet, I. Fisher, J. Garcia-Ojlavo, C. R. Mirasso, L. Pesquera, and A. Shore, "Chaos-based communications at high bit rates using commercial fibre-optic links," $\mathrm{Na}$ ture, vol. 437/17, pp. 343-346, 2005.

[3] P. Chargé, D. Fournier-Prunaret, and V. Guglielmi, "Features analysis of a parametric PWL chaotic map and its utilization for secure transmissions," Chaos, Solitons and Fractals, vol. 38, pp. 1411-1422, 2008.

[4] C. C. Chen, K. Yao, K. Umeno, and E. Biglieri, "Design of spread-spectrum sequences using chaotic dynamical systems and ergodic theory," IEEE Transactions Circuits and Systems -1: Fundamental Theory and Applications, vol. 48, pp. 1110-1114, 2001.

[5] J. Cheng and N. C. Beaulieu, "Accurate DS-CDMA bit-error probability calculation in Raleigh fading," IEEE Transactions on Wireless Communications, vol. 1, pp. 3-15, 2002.

[6] H. Dedieu, M. P. Kennedy, and M. Hasler, "Chaos shift keying: modulation and demodulation of a chaotic carrier using self-synchronizing Chua's circuits," IEEE Transactions on Circuits and Systems-II: Analog and Digital Signal Processing, vol. 40, pp. 634-642, 1993.

[7] R. Eposito, "Error probabilities for the Nakagami channel," Proceedings IEEE Transactions Information Theory, vol. 13, pp. 145-148, 1967. 
[8] G. Kaddoum, P. Charge, D. Roviras, and D. Fournier-Prunaret, "Comparison of chaotic sequences ina chaos based DS-CDMA system," in International Symposium on Nonlinear Theory and its Applications (NOLTA). Vancouver, Canada, 2007.

[9] M. P. Kennedy, R. Rovatti, and G. Setti, Chaotic Electronics in Telecommunications. London: CRC Press, 2000.

[10] T. Kohda and A. Tsuneda, "Pseudonoise sequences by chaotic nonlinear mapsand their correlation properties," IEICE Transactions Communications, vol. E76-B, pp. 855-862, 1993.

[11] G. Kolumban, B. Vizvari, W. Schwarz, and A. Abel, "Differential chaos shift keying: a robust coding for chaotic communication," presented at Nonlinear Dynamics of Eletronic Systems, Seville, Spain, 1996.

[12] G. Kolumban, G. Kis, Z. Jako, and M. P. Kennedy, "FM-DCSK: a robust modulation scheme for chaos communication," IEEE Transactions on Fundamentals of Electronics, Communications and Computer Sciences, vol. 81, pp. 1798-1802, 1998.

[13] G. K. Kolumban, "Theoretical noise performance of correlator-based chaotic communications schemes," IEEE Transactions on Circuits and Systems-1: Fundamental Theory and Applications, vol. 47, pp. 1692-1701, 2000.

[14] L. E. Larson, J.-M. Liu, and L. S. Tsimring, "Digital Communications Using Chaos and Nonlinear Dynamics." New York: Springer, 2006.

[15] F. C. M. Lau and C. K. Tse, Chaos-based Digital Communications Systems. Heidelberg: Springer Verlag, 2003.

[16] A. J. Lawrance and N. Balakrishna, "Statistical aspects of chaotic maps with negative dependence in a communications setting," Journal of the Royal Statistical Society, Series B, vol. 63, pp. 843-853, 2001.

[17] A. J. Lawrance and G. Ohama, "Exact calculation of bit error rates in communication systems with chaotic modulation," IEEE Transactions on Circuits and Systems I: Fundamental Theory and Applications, vol. 50, pp. 13911400, 2003.

[18] A. J. Lawrance and T. Papamarkou, "Higher order dependency of chaotic maps," in Nonlinear Theory and its Applications 2006 (NOLTA2006). Bologna, Italy, 2006.

[19] A. J. Lawrance and J. Yao, "Likelihood-based demodulation in multi-user chaos shift keying communication," Circuits, Systems, and Signal Processing, vol. 27, pp. 847-864, 2008.

[20] W. C. Lindsey, "Error probabilities for Rician fading multichannel reception of binary and N-ary signals," IEEE Transactions Information Theory, vol. 10, pp. 339-350, 1964.

[21] J. G. Proakis, Digital Communication. Boston: McGraw Hill, 2001.

[22] T. Schimming and M. Hasler, "Optimal detection of differential chaos shift keying," IEEE Transactions on Circuits and Systems I, vol. 47, pp. 1712-1719, 2000.

[23] P. Stavroulakis, Chaos Applications in Telecommunications. New York: CRC Press, 2006.

[24] M. Sushchik, L. S. Tsimring, and A. R. Volkovskii, "Performance analysis of correlation-based communication schemes utilizing chaos," IEEE Transactions on Circuits and Systems-1: Fundamental Theory and Applications, vol. 47, pp. 1684-1691, 2000.

[25] W. M. Tam, F. C. M. Lau, C. K. Tse, and M. Yip, "An approach to calculating the bit-error rate of a coherent chaos-shift-keying communication system under a noisy multiuser environment," IEEE Transactions on Circuits and Systems - I: Fundamental Theory and Applications, vol. 49, pp. 210-233, 2002.

[26] W. M. Tam, F. C. M. Lau, C. K. Tse, and A. J. Lawrance, "Exact analytical of bit error rates for multiple access chaos-based communication systems. ," IEEE Transactions on Circuits and Systems: Part II, vol. 51, pp. 473481, 2004.

[27] W. M. Tam, F. C. M. Lau, and C. K. Tse, Digital Communications with Chaos. Oxford: Elsevier, 2007.

[28] A. Uchida, S. Yoshimori, M. Shinozuka, T. Ogawa, and F. Kannari, "Chaotic on-off keying for secure communications," Optics Letters, vol. 26, pp. 866-868, 2001.

[29] J. Yao, "Optimal chaos shift keying communications with correlation decoding," in IEEE International Symposium on Circuits and Systems (ISCAS2004), vol. IV. Vancouver, Canada, 2004, pp. 593-596.

[30] J. Yao and A. J. Lawrance, "Bit error rate calculation for multi-user coherent chaos-shift-keying communication systems," IEICE Transactions Fundamentals, vol. E87-A, pp. 2280-2291, 2004. 